\title{
CHARACTERIZATION OF A 6-KM DAMPING RING FOR THE INTERNATIONAL LINEAR COLLIDER*
}

\author{
A. Xiao", FNAL, Batavia, IL 60510, U.S.A. \\ L. Emery, ANL, Argonne, IL 60439, U.S.A.
}

\section{Abstract}

Several damping ring designs for the International Linear Collider (ILC) have been proposed recently [1]. One particular design has a circumference of $\sim 6 \mathrm{~km}$ (hoping to take advantage of future kicker technology advances) using TME arc cells and $77 \mathrm{~m}$ of $2 \mathrm{~T}$ wigglers. In this paper, we report beam dynamics characterizations and optimizations for this design. We used the accelerator code elegant [2] for matching and tracking and a 100CPU Linux cluster to provide high throughput.

\section{INTRODUCTION}

A damping ring is one of the essential parts of the proposed ILC project. The basic requirements of the positron ring design shown in Table 1 are specified in the TESLA technical design report [3]. (We mention the positron ring because it's much more difficult than electron ring due to large injected beam emittance). To damp the $1 \mathrm{~ms}$ main linac bunch train at once, the damping ring has to work in a compressed mode. Based on the assumption of achievable kicker ability, a $6 \mathrm{~km}$ damping ring lattice was initially proposed at FNAL [4].

Table 1: Some requirements for ILC $\mathrm{e}^{+}$Damping Ring

\begin{tabular}{|l|c|}
\hline Injected emittance $\gamma \mathcal{E}_{x(y)}$ & $0.01 \mathrm{~m}-\mathrm{rad}$ \\
\hline Extracted emittance $\gamma \varepsilon_{x} / \gamma \mathcal{E}_{y}$ & $8 \times 10^{-6} / 2 \times 10^{-8} \mathrm{~m}$-rad \\
\hline Damping time $\tau_{d}$ & $28 \mathrm{~ms}$ \\
\hline Pulse length $\tau_{\text {train }}$ & $1 \mathrm{~ms}$ \\
\hline Number of bunches $n_{b}$ & 2820 \\
\hline Particles per bunch $N_{e}$ & $2 \times 10^{10}$ \\
\hline
\end{tabular}

To reach the very low extracted beam emittance and short damping time, the ring has to be strongly focused with long wiggler sections. Add to this a large injection beam emittance, the dynamic aperture becomes a very difficult issue. For the required ILC charge and repetition rate the average injected beam power for a $5 \mathrm{GeV}$ ring will be $226 \mathrm{~kW}$, so even loss a small fraction of the injected beam can cause large radiation loads in the damping ring, a concern pointed out in [5].

In this paper, we describe the changes from the original FNAL design, the optimization of the dynamic aperture by taking the advantage of elegant and a 100-CPU Linux cluster at APS, and then specify a new lattice based on a finding from an "abstract" model. Sample particles

\footnotetext{
* Work supported by U.S. Department of Energy, Office of Basic Energy Sciences under Contract No. W-31-109-ENG-38.

\#xiaoam@fnal.gov
}

from positron source simulation have been used for testing the dynamic aperture. We tracked them for one damping time with/without RF and damping effects. All particles survived through tracking.

\section{OPTIMIZATION OF DYNAMIC APERTURE USING ABSTRACT LATTICE}

The dynamic aperture and tune footprint of the original design for different amplitude of particles are shown in Figure 1. Even though the tracking results shows that we have about 6x7 sigma of injection beam dynamic aperture, the real machine will not because the particles that cross the half integer resonance line will be lost. This situation has to be improved significantly.
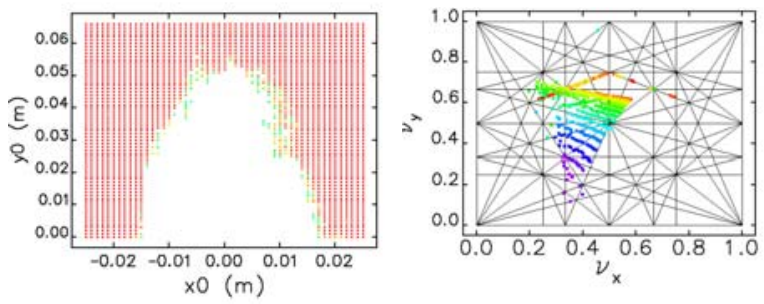

Figure 1: Dynamic aperture and footprint of original design (aperture scale is $\pm 10 \times 10$ sigma of injected beam)

In practical experience, we know that dynamic aperture strongly depends on several parameters: lattice structure; sextupole strength; tunes; phase advance at straight section, etc. To optimize the dynamic aperture, we performed parameter scans of the above.

\section{Abstract Lattice Model}

Parameters scan with a fully developed lattice is very cumbersome and obtaining a reasonable matched lattice is not guaranteed for all parameter sets. To avoid such difficulty an "abstract" lattice model has been used. This lattice is composed with normal TME arc cells and a matrix-type element for the straight sections. Only the matrix element need to be changed when scanning a parameter such as the phase advance of straight sections,.

TCl scripts were written to handle all the tasks: setting up a new parameter set; calculating matrix elements for the chosen parameter sets; writing new lattice files on disk; evoking elegant with the new lattice file; submitting a job to the 100-CPU Linux cluster for tracking large number of particles; processing the results and writing them to separate files.

Before the parameter scans, we designed our TME cells as a basic lattice structure. The cell length, phase advance and total number of cells were chosen so that if we used them to compose a real lattice, the cells would provide 
correct emittance and damping time. To cancel the second-order geometric aberrations we chose two types of TME cells with horizontal/vertical phase advances of $150^{\circ} / 90^{\circ}$ and $135^{\circ} / 90^{\circ}$. The minimum number of cells per arc is $12(2.5 \pi$ per 6 cells $)$ for the $150^{\circ}$ lattice and $8(1.5 \pi$ per 4 cells) for $135^{0}$ lattice. Figure 2 shows the optical functions for $150^{\circ} \mathrm{TME}$ cell.

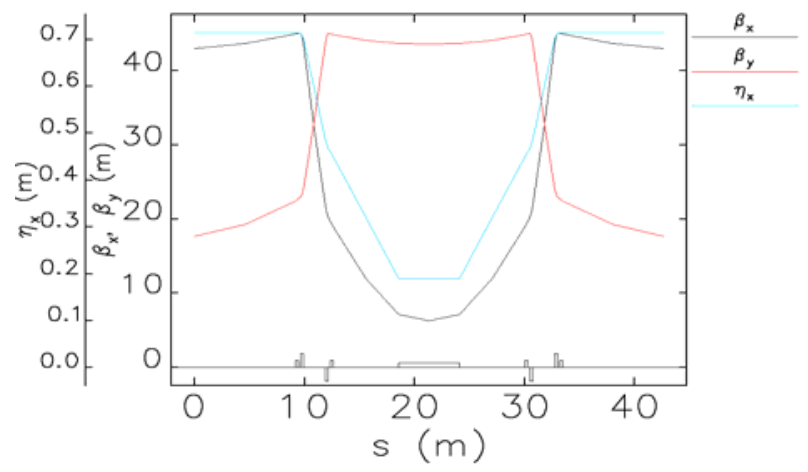

Figure 2: Optical functions of normal TME cell $\left(150^{\circ}\right)$

\section{Parameter scan}

The TME cells above were grouped into 2, 6 or 12 arcs to approximate a scan on the number of superperiods (NP) of the ring. Tunes for each straight section were varied from 0 to 0.5 . For each tune sets $v_{x}$ and $v_{y}$, we launched a grid of $27 \times 34$ particles covering $\pm 20 \times 20 \sigma_{\text {inj. }}$. We tracked these particles for 500 turns and counted the number of surviving particles. Figure 3 shows the contour plots of number of surviving particles in a $v_{x} \times v_{y}$ plane for different NP.

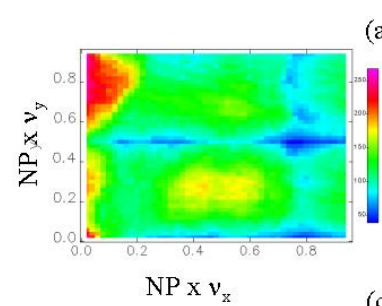

(a)
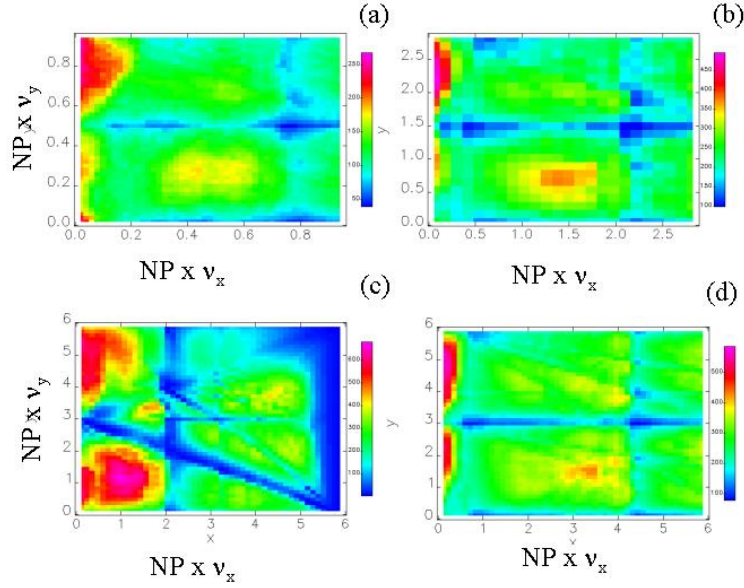

c)

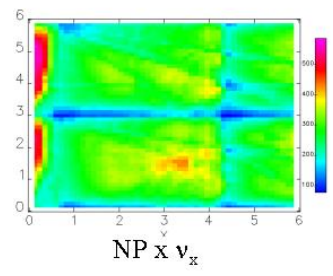

Figure 3: Total surviving particles in two-dimensional straight section phase advance scan for $150^{\circ} \mathrm{TME}$ cells abstract lattice. (a) $\mathrm{NP}=2$; (b) $\mathrm{NP}=6$; (c) $\mathrm{NP}=12$, (d) $\mathrm{NP}=12 \quad\left(135^{0}\right.$ lattice $) . \mathrm{X}$-axis is $N P \times v_{x}, \mathrm{Y}$-axis is $N P \times v_{y}$

The following conclusions can be drawn from Figure 3:

- More superperiods give better dynamic aperture. Only the structure resonance lines are dangerous.

- Phase advance for straight sections is the dominating effect for dynamic aperture.
- The total phase advance (tune) has not much effect on dynamic aperture compared with the first two effects.

- Phase advance solution for straight section is not obviously $\pi$ or $2 \pi$. The solution depends on arc cell structure. Roughly the end sextupoles in the arcs should be separated by an odd multiple of $\pi$.

The dynamic aperture for the best parameter set is shown in Figure 4. The wiggler effects and chromaticity from straight sections have been turned off.

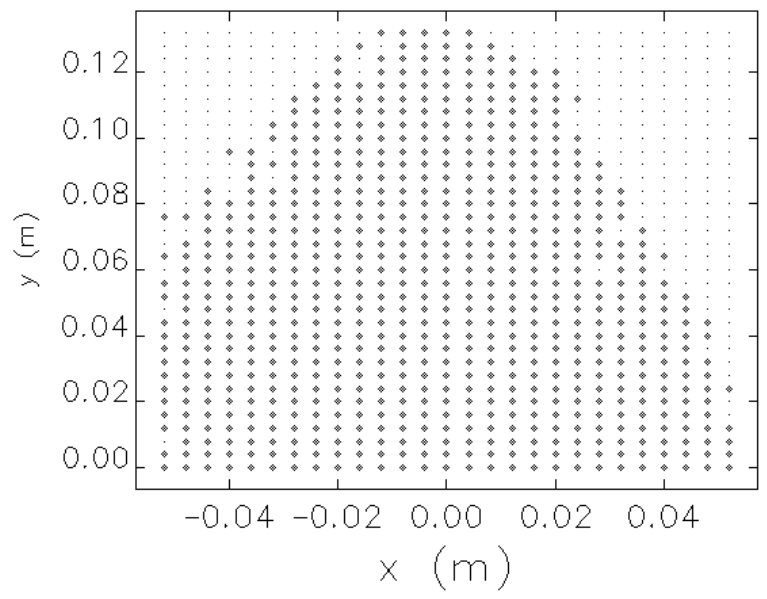

Figure 4: Dynamic aperture for optimized abstract lattice (the window size is $\pm 20 \times 20 \sigma_{\text {inj }}$ )

\section{A 6-KM DAMPING RING BASED ON THE OPTIMIZED ABSTRACT LATTICE}

Based on the optimization results from the abstract lattice model, we decided that our new lattice for ILC damping ring should have 12 periods. There are two considerations on selecting the TME cell type $\left(135^{\circ}\right.$ or $\left.150^{\circ}\right)$ : the circumference and the occupancy rate of the arcs. The circumference is constrained by the injection and extraction scheme. The straight sections have to be long enough for injection/extraction equipment, wigglers, $\mathrm{RF}$ and others, but not too long, because this will increase the strength of chromaticity sextupoles in the arcs. In the end, we chose the $135^{\circ}$ cell. The optical functions of the ring are shown in Figure 5.

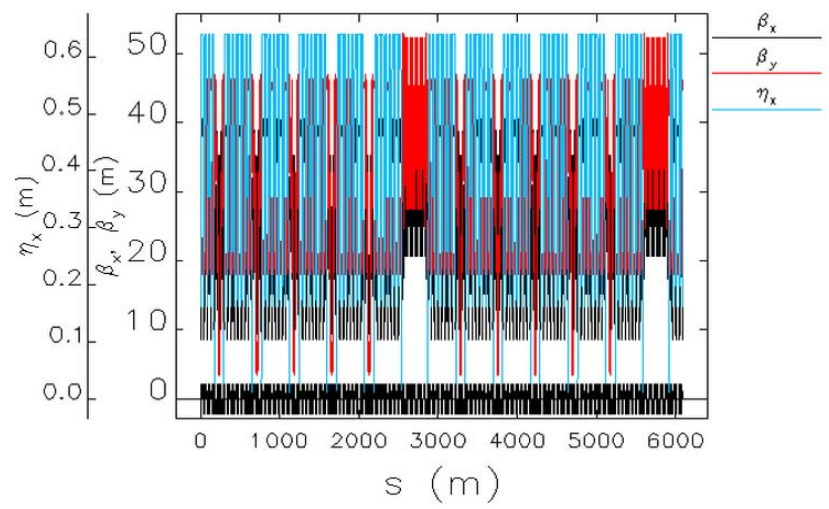

Figure 5: Optical functions of 6-km ILC damping ring 
The ring's general lattice parameters are summarized in Table 2. We have 10 straight sections devoted to the wigglers and 2 long straight sections for injection, extraction and RF.

Table 2: General Lattice Parameters

\begin{tabular}{|l|c|}
\hline Energy E & $5 \mathrm{GeV}$ \\
\hline Circumference C & $6113.967 \mathrm{~m}$ \\
\hline Horizontal Emittance $\varepsilon_{\mathrm{x}}$ & $0.67 \mathrm{~nm}-\mathrm{rad}$ \\
\hline Damping Time $\tau_{\mathrm{d}}$ & $27.5 \mathrm{~ms}$ \\
\hline Tunes $v_{\mathrm{x}}, v_{\mathrm{y}}, v_{\mathrm{s}}$ & $50.96,40.92,0.043$ \\
\hline Natural Chromaticity $\xi_{\mathrm{x}}, \xi_{\mathrm{y}}$ & $-65.42,-55.25$ \\
\hline Momentum Compaction Factor $\alpha_{\mathrm{p}}$ & $1.59 \mathrm{E}-4$ \\
\hline Bunch Length $\sigma_{\mathrm{z}}$ & $5.6 \mathrm{~mm}$ \\
\hline Energy Spread $\delta \mathrm{p} / \mathrm{p}$ & $0.153 \%$ \\
\hline $\mathrm{V}_{\mathrm{RF}}$ & $36 \mathrm{MV}$ \\
\hline Energy Loss per Turn $\mathrm{U}_{0}$ & $7.5 \mathrm{MeV}$ \\
\hline RF Acceptance $\delta_{\mathrm{RF}}$ & $4.4 \%$ \\
\hline
\end{tabular}

\section{DYNAMIC APERTURE AND PARTICLE TRACKING}

There are two families of sextupoles in the lattice; they are tuned to give zero chromaticity for on-momentum particles. The remaining tune-shifts with energy are illustrated in Figure 6. The dynamic aperture of the new lattice is shown in Figure 7 together with the footprint of on-momentum particles. The aperture for the new design is larger than the old one, and the tune no longer crosses the half integer resonance line. The aperture for offmomentum particles shown here indicates that we have a good dynamic aperture for energy errors.
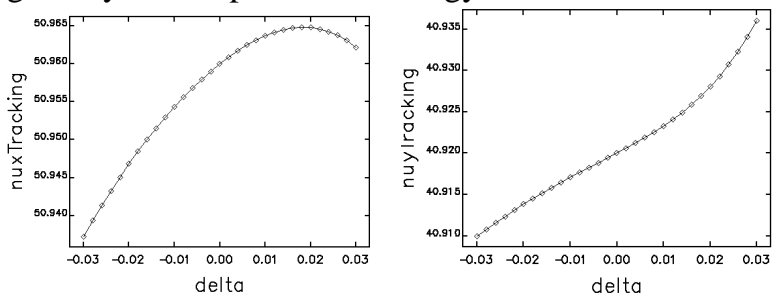

Figure 6: Tune shift with energy
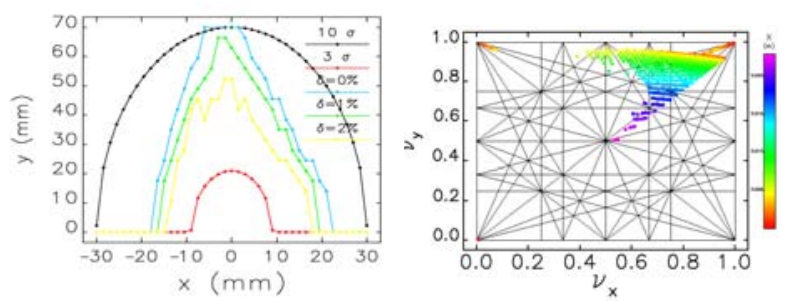

Figure 7: Dynamic aperture and tune footprint for the optimized lattice

To test the new lattice with a realistic positron beam, we tracked a sample particles distribution produced by Y.
Batygin [1]. The normalized emittances of sample particles are about $0.006 \mathrm{~m}$-rad in horizontal and 0.0068 $\mathrm{m}$-rad in vertical with a 0.003 energy spread. We tracked the 1980 sample particles for 1 damping time (1380 turns) with linear wiggler effects, and had no particle loss. The injected phase space and the phase space after tracking are shown in Figure 8.
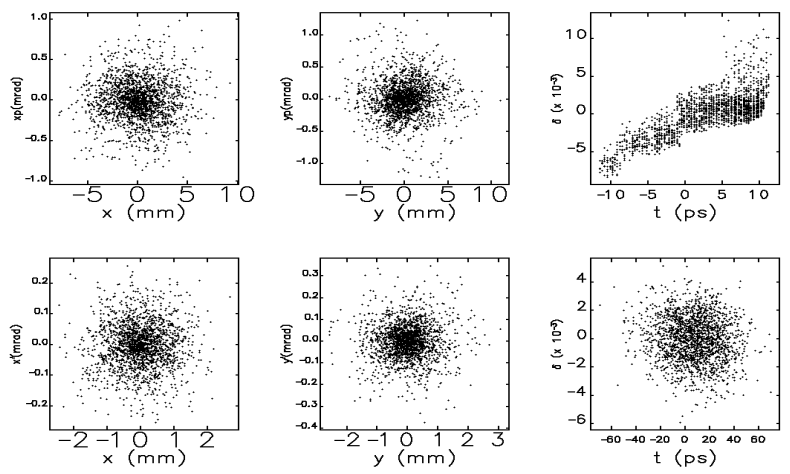

Figure 8: Phase Space of Sample Particles before tracking (top) and after tracking (bottom)

\section{SUMMARY AND CONCLUSIONS}

We based our dynamic aperture optimization on an abstract lattice. This abstract lattice is composed of basic arc cells (TME in our case) and matrix elements for simulating straight sections. The dynamic aperture for the abstract lattice model had been optimized through parameter scans. A new lattice was built upon the findings of the abstract model. We studied the chromaticity and dynamic aperture properties for the new lattice then tracked with a realistic particle distribution from a positron source simulation. The results are very encouraging. It's reasonable to believe that the same method will be valid with other lattice designs in general and would act as a guideline for choosing lattice configuration.

\section{ACKNOWLEDGEMENT}

We want to thank Michael Borland of APS for helping us with elegant and Vadim Sajaev of APS for the useful discussion on how to avoid machine resonance.

\section{REFERENCES}

[1] ILC Damping Ring Collaboration, www.desy.de/ awolski/ILCDR/

[2] M. Borland, "elegant: A Flexible SDDS-Compliant Code for Accelerator Simulation," Advanced Photon Source LS-287, September 2000.

[3] TESLA Technical Design Report, DESY 2001-011 (March 2001).

[4] S. Mishra et al, "Studies Pertaining to a Small Damping Ring for the International Linear Collider," FNAL Report (October 2004).

[5] A. Wolski, "Lattices with Large Dynamic Aperture for ILC Damping Rings,” LBNL-57045. 